\title{
Seroprevalence of Erythrovirus B19 IgG Antibody among Paediatric Patients in Makkah and Jeddah, Kingdom of Saudi Arabia
}

\author{
Ayman Johargy \\ Department of Microbiology, Faculty of Medicine, Umm Al-Qura University, Makkah, Saudi Arabia
}

\section{Key Words}

Makkah • Saudi Arabia • Paediatric patients - Erythrovirus

B19 • Enzyme-linked immunosorbent assay

\begin{abstract}
Objective: To estimate the prevalence of $\lg G$ antibodies against B19 virus (B19V) in Makkah and Jeddah, Saudi Arabia. Methods: B19V-specific IgG antibodies were detected by a commercial indirect enzyme-linked immunosorbent assay in sera of 400 paediatric patients (185 males and $215 \mathrm{fe}-$ males) aged 1-17 years. Results: Of the 400 patients, 80 (20\%) had sera positive for B19V-specific IgG. The difference in the prevalence of the antibodies between genders was not statistically significant $(p=0.9)$. The prevalence of antiB19V antibodies increased significantly in the age group of $12-17$ years as compared to younger patients ( 37.5 vs. $18 \%$ in those aged $1-11$ years; $p=0.006)$. Conclusion: This study indicated a high prevalence of IgG antibodies against B19V in paediatric patients with an increase in age.
\end{abstract}

Copyright $\odot 2009$ S. Karger AG, Basel

\section{Introduction}

The B19 virus (B19V), also known as parvovirus B19, is a member of the genus Erythrovirus within the Parvoviridae family. It was discovered by chance in 1974 by Yvonne Cossart in England during routine screening for hepatitis B of asymptomatic blood donors [1]. The most common clinical manifestation of $\mathrm{B} 19 \mathrm{~V}$ infection is erythema infectiosum (also called fifth disease or 'slappedcheek' syndrome), which is characterised by a facial rash that spreads to the trunk and limbs, usually preceded by a non-specific flu-like illness. B19V is also associated with arthropathy, aplastic crisis, chronic pure red cell aplasia and foetal infection. Less commonly, B19V may be associated with myocarditis, various vasculitic syndromes and neurological disorders. B19V is a ubiquitous virus that tends to produce spring epidemics in temperate climates in children $4-10$ years of age [2].

Infection with $\mathrm{B} 19 \mathrm{~V}$ is thought to confer lifelong immunity. The most common method for detection of $\mathrm{B} 19 \mathrm{~V}$-specific antibodies is enzyme-linked immunosorbent assay (ELISA). Specific IgM antibodies are considered to be serological markers for primary B19V infection. IgG antibodies against B19V are produced approximately 2 weeks after infection and persist for life. The presence of these antibodies is indicative of past infection [3] as determined for various parts of the world [4].

\section{KARGER \\ Fax +41613061234 \\ E-Mail karger@karger.ch}

www.karger.com
(C) 2009 S. Karger AG, Basel

$1011-7571 / 09 / 0184-0339 \$ 26.00 / 0$

Accessible online at:

www.karger.com/mpp
Ayman Johargy, Assistant Professor of Molecular Virology

Head of Microbiology Department and Head of Medical Research Centre

Faculty of Medicine, Umm Al-Qura University

Makkah (Saudi Arabia)

Tel./Fax +966 2527 0446, E-Mail johargy@hotmail.com 
The aim of this study was to determine the prevalence of B19V-specific IgG antibodies in children from Makkah and Jeddah of Saudi Arabia.

\section{Patients and Methods}

An informed consent was obtained from the parents of each child before inclusion in the study. Parents of the participating children were informed about the procedure before blood samples were collected from their children. Four hundred paediatric patients (selected by simple randomization) were included in the study as follows: 140 from the Maternity and Children's Hospital, Makkah; 95 from the Maternity and Children's Hospital, Jeddah; 65 from the King Abdul-Aziz General Hospital, Jeddah; 30 from the Al-Noor Hospital, Makkah; 25 from the Bakhsh Hospital, Makkah; 25 from the King Fahad General Hospital, Jeddah, and 20 from the Hera Hospital, Makkah. One hundred and fifty came for vaccination, while the remaining 250 were hospitalized for various pathological symptoms. The study was carried out from November 2006 to November 2007. The age range of the patients was $1-17$ years, with a mean age of $6.48 \pm 4$ years, median age of 6 years and mode of 9 years.

A sample of $10 \mathrm{ml}$ of blood was collected by venepuncture from each subject; serum was separated, aliquoted into 2 Eppendorf tubes and stored at $-20^{\circ} \mathrm{C}$ until testing. Sera were tested for the presence of IgG antibodies to B19V using a commercial ELISA (Novatec; Immunodiagnostic GmbH, Germany, Distributor: Dia Sorin, Italy). The recombinant Novatec B19V IgG ELISA offers increased diagnostic specificity and sensitivity by employing the highly purified antigens VP-1S, VP-C and VP-N. According to the manufacturer, diagnostic sensitivity and specificity of the test were greater than 95\%. Cut-off values were calculated according to the manufacturer's instructions, where the cut-off was the mean absorbance value of the cut-off control determinations. Samples were considered positive if the absorbance was higher than $10 \%$ over the cut-off.

The prevalence was statistically analysed by calculating the mean, median, standard deviation, mode, range and $p$ value, and distributed according to age and gender using a Fisher test (Graph Pad Instat programme); $p$ values $<0.05$ were considered significant. Prevalence estimates in this study required statistical evaluation: males versus females, age group 1-11 versus age group 1217 and age group $1-5$ versus age group $6-11$.

\section{Results}

Of the 400 paediatric patients, $80(20 \%)$ were positive while the remaining $320(80 \%)$ were negative. Of the 185 males, 36 (19.5\%) were positive for B19V-specific IgG while of the 215 females 44 (20.5\%) were positive. However, the difference between the genders was not statistically significant $(\mathrm{p}=0.9)$. The seroprevalence with regard to age distribution is shown in table 1 .
Table 1. Seroprevalence of B19V-specific IgG antibody among different age categories

\begin{tabular}{llll}
\hline Age category & Positive & Negative & Total \\
\hline $1-5$ years & $31(16.8 \%)$ & $154(83.2 \%)$ & $185(100 \%)$ \\
$6-11$ years & $34(19.4 \%)$ & $141(80.6 \%)$ & $175(100 \%)$ \\
$12-17$ years & $15(37.5 \%)$ & $25(62.5 \%)$ & $40(100 \%)$ \\
\hline Total & 80 & 320 & 400 \\
\hline
\end{tabular}

Clearly, in an age-dependent manner, the prevalence increased significantly from $18 \%$ at $1-11$ years to $37.5 \%$ at $12-17$ years $(\mathrm{p}=0.006)$ indicating age dependence. However, the difference in prevalence of $\mathrm{B} 19 \mathrm{~V}$-specific IgG antibody between age group $1-5(16.8 \%)$ and $6-11$ years $(19.4 \%)$ was not statistically significant $(\mathrm{p}=0.6)$.

\section{Discussion}

The 20\% anti-B19V IgG seroprevalence as determined in our study for Jeddah and Makkah is consistent with an overall prevalence of $19.0 \%$ previously reported for the Kingdom of Saudia Arabia [5] but slightly higher than the $17 \%$ reported in neighbouring Kuwait [6]. However, our findings were lower than those of Cohen and Buckley [3] assessed in England and Wales where the B19V seroprevalence in children ( $0-10$ years) was $27 \%$, which might be explained by more exposure to the $\mathrm{B} 19 \mathrm{~V}$ in these countries (England and Wales) due to early ages of school entry.

In our study also, the B19V seroprevalence significantly increased from $18 \%$ at $1-11$ years to $37.5 \%$ at $12-17$ years, suggesting that infection principally occurs during the school years. This interpretation is supported by a study by Valeur-Jensen et al. [7] from Denmark which concluded that the highest risk of $\mathrm{B} 19 \mathrm{~V}$ infection is associated with having school-aged children in the house and that the susceptibility to B19V infection increases with the number of school-aged children per family.

\section{Conclusion}

This study indicated a high prevalence of IgG antibodies against $\mathrm{B} 19 \mathrm{~V}$ in the paediatric patients associated with an increase in age of the 1- to 17-year-old children in Makkah and Jeddah of Saudi Arabia. 


\section{References}

-1 Cossart YE, Field AM, Cant B, Widdows D: Parvovirus-like particles in human sera. Lancet 1975;i:72-73.

$\checkmark 2$ Young NS, Brown KE: Parvovirus B19. N Engl J Med 2004;350:586-597.

$\checkmark 3$ Cohen BJ, Buckley MM: The prevalence of antibody to human parvovirus B19 in England and Wales. J Med Microbiol 1988;25: 151-153.
-4 Kelly HA, Siebert D, Hammond R, Leydon J, Kiely P, Maskill W: The age-specific prevalence of human parvovirus immunity in Victoria, Australia, compared with other parts of the world. Epidemiol Infect 2000;124: 449-457.

5 al Frayh AR, Bahakim H, Kidess E, Ramia S: IgG and IgM antibodies to human parvovirus B19 in the serum of patients with a clinical diagnosis of infection with the virus and in the general population of Saudi Arabia. J Infect 1993;27:51-55.
-6 Alsaeid K, Alsaeid M, Essa S, Dimitrov D, Pacsa A: Seroprevalence of human parvovirus B19 in children of a desert region. Ann Trop Paediatr 1996;16:255-257.

7 Valeur-Jensen AK, Pedersen CB, Westergaard T, Jensen IP, Lebech M, Andersen PK, Aaby P, Pedersen BN, Melbye M: Risk factors for parvovirus B19 infection in pregnancy. JAMA 1999;281:1099-1105. 